\title{
Capacity Bounds on the Downlink of Symmetric, Multi-Relay, Single Receiver C-RAN Networks
}

\author{
Shirin Saeedi Bidokhti, Gerhard Kramer and Shlomo Shamai (Shitz)
}

\begin{abstract}
The downlink of symmetric Cloud Radio Access Networks (C-RANs) with multiple relays and a single receiver is studied. Lower and upper bounds are derived on the capacity. The lower bound is achieved by Marton's coding which facilitates dependence among the multiple-access channel inputs. The upper bound uses Ozarow's technique to augment the system with an auxiliary random variable. The bounds are studied over scalar Gaussian C-RANs and are shown to meet and characterize the capacity for interesting regimes of operation.
\end{abstract}

\section{INTRODUCTION}

Cloud Radio Access Networks (C-RANs) are expected to be a part of future mobile network architectures. In CRANs, information processing is done in a cloud-based central unit that is connected to remote radio heads (or relays) by rate-limited fronthaul links. C-RANs improve energy and bandwidth efficiency and reduce complexity of relays by facilitating centralized information processing and cooperative communication. We refer to [1]-[3] and the references therein for an overview of the challenges and coding techniques for C-RANs. Several coding schemes have been proposed in recent years for the downlink of C-RANs including message sharing [4], backhaul compression [5], hybrid schemes [6], and generalized data sharing using Marton's coding [7], [8]. While none of these schemes are known to be optimal, [9] has proved an upper bound on the sum-rate of 2-relay C-RANs with two users and numerically compared the performance of the aforementioned schemes with the upper bound.

We consider the downlink of a C-RAN with multiple relays and a single user. This network may be modeled by an $M$ relay diamond network where the broadcast component is modeled by rate-limited links and the multiaccess component is modeled by a memoryless multiple access channel (MAC), see Fig. 1. The capacity of this class of networks is not known in general, but lower and upper bounds were derived in [10]-[12] for 2-relay networks. Moreover, the capacity was found for binary adder MACs [12], and for certain regimes of operation in Gaussian MACs [11], [12]. In this work, we derive lower and upper bounds for symmetric C-RANs with multiple relays and find the capacity in interesting regimes of operation for symmetric Gaussian C-RANs.

S. Saeedi Bidokhti is with the Department of Electrical Engineering at Stanford University, USA. G. Kramer is with the Department for Electrical and Computer Engineering, Technical University of Munich, Germany. $\mathrm{S}$. Shamai is with the Department of Electrical Engineering, Technion, Israel (saeedi@stanford.edu,gerhard.kramer@tum.de,sshlomo@ee.technion.ac.il).

The work of S. Saeedi Bidokhti was supported by the Swiss National Science Foundation fellowship no. 158487. The work of G. Kramer was supported by the German Federal Ministry of Education and Research in the framework of the Alexander von Humboldt-Professorship. The work of S. Shamai was supported by the European Union's Horizon 2020 Research and Innovation Program, grant agreement no. 694630.

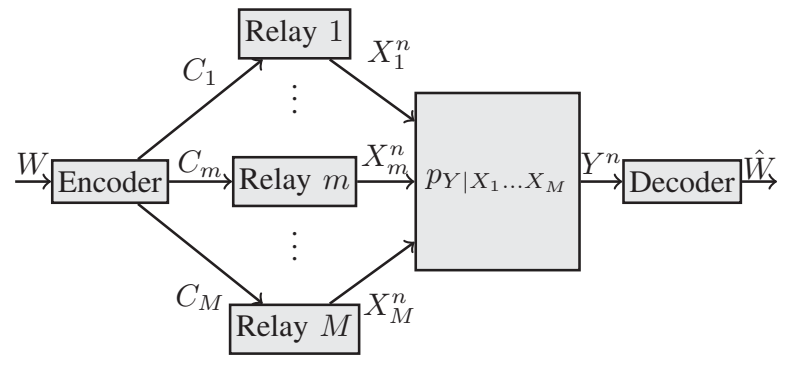

Fig. 1: A C-RAN downlink.

The rest of the paper is organized as follows. Section II introduces notation and the problem setup. In Section III, we propose a coding strategy based on Marton's coding and discuss simplifications for symmetric networks. In Section IV, we generalize the bounding technique in [11], [12]. The case of Gaussian C-RANs is studied in Section V, where we compute lower and upper bounds and show that they meet in certain regimes of operation characterized in terms of power, number of users, and broadcast link capacities.

\section{Preliminaries and Problem Setup}

\section{A. Notation}

Random variables are denoted by uppercase letters, e.g. $X$, their realizations are denoted by lowercase letters, e.g. $x$, and their corresponding probabilities are denoted by $p_{X}(x)$ or $p(x)$. The probability mass function (pmf) describing $X$ is denoted by $p_{X} . \mathcal{T}_{\epsilon}^{n}(X)$ denotes the set of sequences that are $\epsilon$-typical with respect to $P_{X}$ [13, Page 25]. When $P_{X}$ is clear from the context we write $\mathcal{T}_{\epsilon}^{n}$. The entropy of $X$ is denoted by $H(X)$, the conditional entropy of $X$ given $Y$ is denoted by $H(X \mid Y)$ and the mutual information between $X$ and $Y$ is denoted by $I(X ; Y)$. Similarly, differential entropies and conditional differential entropies are denoted by $h(X)$ and $h(X \mid Y)$.

Matrices are denoted by bold letters, e.g. K. We denote the entry of matrix $\mathbf{K}$ in row $i$ and column $j$ by $\mathbf{K}_{i j}$. Sets are denoted by script letters, e.g., $\mathcal{S}$. The cartesian product of $\mathcal{S}_{1}$ and $\mathcal{S}_{2}$ is denoted by $\mathcal{S}_{1} \times \mathcal{S}_{2}$, and the $n$-fold Cartesian product of $\mathcal{S}$ is denoted by $\mathcal{S}^{n}$. The cardinality of $\mathcal{S}$ is denoted by $|\mathcal{S}|$.

Given the set $\mathcal{S}=\left\{s_{1}, \ldots, s_{|\mathcal{S}|}\right\}, X_{\mathcal{S}}$ denotes the tuple $\left(X_{s_{1}}, \ldots, X_{s_{|\mathcal{S}|}}\right)$. The random string $X_{1}, \ldots, X_{n}$ is denoted by $X^{n} \cdot I\left(X_{\mathcal{S}}\right)$ is defined as follows (see [14, Eqn (74)]):

$$
I\left(X_{\mathcal{S}}\right)=\sum_{m \in \mathcal{S}} H\left(X_{m}\right)-H\left(X_{\mathcal{S}}\right) .
$$

For example, when $\mathcal{S}=\left\{s_{1}, s_{2}\right\}$, (1) becomes the mutual information $I\left(X_{s_{1}} ; X_{s_{2}}\right)$. The conditional version of (1), 
$I\left(X_{\mathcal{S}} \mid U\right)$, is defined similarly by conditioning all terms in (1) on $U$. Note that $I\left(X_{\mathcal{S}} \mid U\right)$ is non-negative.

\section{B. Model}

Consider the C-RAN in Fig. 1, where a source communicates a message $W$ with $n R$ bits to a sink with the help of $M$ relays. Let $\mathcal{M}=\{1, \ldots, M\}$ be the set of relays.

The source encodes $W$ into descriptions $\mathbb{V}_{1}, \ldots, \mathbb{V}_{M}$ that are provided to relays $1, \ldots, M$, respectively. We focus mainly on symmetric networks where $\mathbb{V}_{m}$ satisfies

$$
H\left(\mathbb{V}_{m}\right) \leq n C, \quad m=1, \ldots, M
$$

Each relay $m, m=1, \ldots, M$, maps its description $\mathbb{V}_{m}$ into a sequence $X_{m}^{n}$ which is sent over a multiple access channel. The MAC is characterized by the input alphabets $\mathcal{X}_{1}, \ldots, \mathcal{X}_{M}$, the output alphabet $\mathcal{Y}$, and the transitional probabilities $p\left(y \mid x_{1}, \ldots, x_{M}\right)$ for all $\left(x_{1}, \ldots, x_{M}, y\right) \in \mathcal{X}_{1} \times \ldots \times \mathcal{X}_{M} \times \mathcal{Y}$. From the received sequence $Y^{n}$, the sink decodes an estimate $\hat{W}$ of $W$.

A coding scheme consists of an encoder, $M$ relay mappings, and a decoder, and is said to achieve the rate $R$ if, by choosing $n$ sufficiently large, we can make the error probability $\operatorname{Pr}(\hat{W} \neq W)$ as small as desired. We are interested in characterizing the largest achievable rate $R$. We refer to the maximum rate as the capacity $C^{(M)}$ of the network.

In this work, we focus on symmetric networks:

Definition 1. The network in Fig. 1 is symmetric if we have

$$
\begin{aligned}
& C_{1}=\ldots=C_{M}=: C \\
& \mathcal{X}_{1}=\ldots=\mathcal{X}_{M}=: \mathcal{X}
\end{aligned}
$$

and

$$
p_{Y \mid X_{1} \ldots X_{M}}\left(y \mid x_{1}, \ldots, x_{M}\right)=p_{Y \mid X_{1} \ldots X_{M}}\left(y \mid x_{1}^{\prime}, \ldots, x_{M}^{\prime}\right)
$$

for all $y \in \mathcal{Y},\left(x_{1}, \ldots, x_{M}\right) \in \mathcal{X}^{M}$ and any of its permutations $\left(x_{1}^{\prime}, \ldots, x_{M}^{\prime}\right)$.

When the MAC is Gaussian, the input and output alphabets are the set of real numbers and the output is given by

$$
Y=\sum_{m=1}^{M} X_{M}+Z
$$

where $Z$ is Gaussian noise with zero mean and unit variance. We consider average block power constraints $P_{1}, \ldots, P_{M}$ :

$$
\frac{1}{n} \sum_{i=1}^{n} \mathbb{E}\left(X_{m, i}^{2}\right) \leq P_{m}, \quad m=1, \ldots, M .
$$

The Gaussian C-RAN is symmetric if $C_{m}=C$ and $P_{m}=P$ for all $m=1, \ldots, M$.

\section{A LOWER BOUND}

We outline an achievable scheme based on Marton's coding. We remark that this scheme can be improved for certain regimes of $C$ by using superposition coding (e.g., see [15, Theorem 2] and [11, Theorem 2]).
Fix the pmf $p\left(x_{1}, \ldots, x_{M}\right), \epsilon>0$, and the auxiliary rates $R_{m}, R_{m}^{\prime}, m=1, \ldots, M$, such that

$$
\begin{aligned}
& R_{m}, R_{m}^{\prime} \geq 0 \\
& R_{m}+R_{m}^{\prime} \leq C_{m} .
\end{aligned}
$$

1) Codebook construction: Set

$$
R=\sum_{m=1}^{M} R_{m}
$$

For every $m=1, \ldots, M$, generate $2^{n\left(R_{m}+R_{m}^{\prime}\right)}$ sequences $x_{m}^{n}\left(w_{m}, w_{m}^{\prime}\right), w_{M}=1, \ldots, 2^{n R_{m}}, w_{M}^{\prime}=1, \ldots, 2^{n R_{m}^{\prime}}$, in an i.i.d manner according to $\prod_{\ell} P_{X_{\ell}}\left(x_{m, \ell}\right)$, independently across $m=1, \ldots, M$. For each bin index $\left(w_{1}, \ldots, w_{M}\right)$, pick a jointly typical sequence tuple

$$
\left(x_{1}^{n}\left(w_{1}, w_{1}^{\prime \star}\right), \ldots, x_{M}^{n}\left(w_{M}, w_{M}^{\prime \star}\right)\right) \in \mathcal{T}_{\epsilon}^{n} .
$$

2) Encoding: Represent message $w$ as a tuple $\left(w_{1}, \ldots, w_{M}\right)$, and send $\left(w_{m}, w_{m}^{\prime \star}\right)$ to relay $m$, $m=1, \ldots, M$.

3) Relay mapping at relay $m, m=1, \ldots, M$ : Relay $m$ sends $X_{m}^{n}\left(w_{m}, w_{m}^{\prime \star}\right)$ over the MAC.

4) Decoding: Upon receiving $y^{n}$, the receiver looks for indices $\hat{w}_{1}, \ldots, \hat{w}_{M}$ for which the following joint typicality test holds for some $\hat{w}_{1}^{\prime}, \ldots, \hat{w}_{M}^{\prime}$ :

$$
\left(x_{1}^{n}\left(\hat{w}_{1}, \hat{w}_{1}^{\prime}\right), \ldots, x_{M}^{n}\left(\hat{w}_{M}, \hat{w}_{M}^{\prime}\right), y^{n}\right) \in \mathcal{T}_{\epsilon}^{n} .
$$

We show in [16, Appendix A] that the above scheme has a vanishing error probability as $n \rightarrow \infty$ if in addition to (8)-(10) we have

$$
\begin{gathered}
\sum_{m \in \mathcal{S}} R_{m}^{\prime} \geq I\left(X_{\mathcal{S}}\right), \quad \forall \mathcal{S} \subseteq \mathcal{M} \\
\sum_{m \in \mathcal{S}} R_{m}+R_{m}^{\prime} \leq I\left(X_{\mathcal{S}} ; Y \mid X_{\overline{\mathcal{S}}}\right)+I\left(X_{\mathcal{M}}\right)-I\left(X_{\overline{\mathcal{S}}}\right), \quad \forall \mathcal{S} \subseteq \mathcal{M} .
\end{gathered}
$$

One can use Fourier-Motzkin elimination to eliminate $R_{m}, R_{m}^{\prime}, m=1, \ldots, M$, from (8)-(10), (13), (14), and characterize the set of achievable rates $R$. For symmetric networks (see Definition 1), we bypass the above step and proceed by choosing $p_{X_{\mathcal{M}}}$ to be "symmetric". We say $p_{X_{\mathcal{M}}}$ is symmetric if

$$
\mathcal{X}_{1}=\ldots=\mathcal{X}_{M}=\mathcal{X}
$$

and for all subsets $\mathcal{S}, \mathcal{S}^{\prime} \subseteq \mathcal{M}$ with $|\mathcal{S}|=\left|\mathcal{S}^{\prime}\right|$ we have

$$
\begin{aligned}
p_{X_{\mathcal{S}}}\left(x_{1}, \ldots, x_{|\mathcal{S}|}\right)=p_{X_{\mathcal{S}^{\prime}}} & \left(x_{1}, \ldots, x_{|\mathcal{S}|}\right), \\
& \forall\left(x_{1}, \ldots, x_{|\mathcal{S}|}\right) \in \mathcal{X}^{|\mathcal{S}|} .
\end{aligned}
$$

We simplify the problem defined by (8)-(10), (13), (14) for symmetric distributions and prove the following result in [16, Appendix B].

Theorem 1. For symmetric $C-R A N$ downlinks, the rate $R$ is achievable if

$$
\begin{aligned}
& R \leq M C-I\left(X_{\mathcal{M}}\right) \\
& R \leq I\left(X_{\mathcal{M}} ; Y\right)
\end{aligned}
$$

for some symmetric distribution $p_{X_{\mathcal{M}}}$. 


$$
C^{(M)} \leq \max _{p(\mathbf{x})} \min _{\substack{p(u \mid \mathbf{x} y) \\
=p(u \mid y)}} \max _{\substack{p \mid \mathbf{x}, u, y) \\
=p(q \mid \mathbf{x})}}\left\{\begin{array}{l}
M C-(M-1) H(U \mid Q)+\sum_{m=1}^{M} H\left(U \mid X_{m} Q\right)-H\left(U \mid X_{\mathcal{M}}\right) \\
\min _{\mathcal{S} \subseteq \mathcal{M}}|\mathcal{S}| C+I\left(X_{\left.\overline{\mathcal{S}} ; Y \mid X_{\mathcal{S}} Q\right)}\right.
\end{array}\right\}
$$

\section{AN UPPER BOUND}

Our upper bound is motivated by [11], [12], [14], [17].

Theorem 2. The capacity $C^{(M)}$ is upper bounded as shown in (19) on the top of this page, where $Q-X_{\mathcal{M}}-Y-U$ forms a Markov chain. Moreover, the alphabet size of $Q$ may be chosen to satisfy $|\mathcal{Q}| \leq \prod_{i=1}^{M}\left|\mathcal{X}_{i}\right|+2^{M}-1$.

Remark 1. For $M=2$, Theorem 2 reduces to [12, Theorem 3].

Proof Outline. We start with the following $n$-letter upper bound (see [16, Appendix C]):

$$
\begin{aligned}
& n R \leq n M C-I\left(X_{\mathcal{M}}^{n}\right) \\
& n R \leq n|\mathcal{S}| C+I\left(X_{\mathcal{M}}^{n} ; Y^{n} \mid X_{\mathcal{S}}^{n}\right), \quad \forall \mathcal{S} \subseteq \mathcal{M} .
\end{aligned}
$$

For any sequence $U^{n}$, we have

$$
\begin{aligned}
I\left(X_{\mathcal{M}}^{n}\right) & \geq I\left(X_{\mathcal{M}}^{n}\right)-I\left(X_{\mathcal{M}}^{n} \mid U^{n}\right) \\
& =\left[\sum_{m=1}^{M} I\left(X_{m}^{n} ; U^{n}\right)\right]-I\left(X_{\mathcal{M}}^{n} ; U^{n}\right) \\
& =(M-1) H\left(U^{n}\right)-\left[\sum_{m=1}^{M} H\left(U^{n} \mid X_{m}^{n}\right)\right]+H\left(U^{n} \mid X_{\mathcal{M}}^{n}\right) .
\end{aligned}
$$

By substituting (22) into (20), we obtain

$$
\begin{aligned}
n R \leq & n M C-(M-1) H\left(U^{n}\right) \\
& +\left[\sum_{m=1}^{M} H\left(U^{n} \mid X_{m}^{n}\right)\right]-H\left(U^{n} \mid X_{\mathcal{M}}^{n}\right) .
\end{aligned}
$$

We now choose $U_{i}, i=1, \ldots, n$, to be the output of a memoryless channel $p_{U \mid Y}\left(u_{i} \mid y_{i}\right)$ with input $Y_{i}$. The auxiliary channel $p_{U \mid Y}(. \mid$.$) will be optimized later. With this choice, we$ single letterize (20) and (21) and obtain

$$
R \leq M C-(M-1) H(U \mid Q)+\sum_{m=1}^{M} H\left(U \mid X_{m} Q\right)-H\left(U \mid X_{\mathcal{M}}\right)
$$

$$
R \leq|\mathcal{S}| C+I\left(X_{\overline{\mathcal{S}}} ; Y \mid X_{\mathcal{S}} Q\right), \quad \forall \mathcal{S} \subseteq \mathcal{M} .
$$

Details of the proof are presented in [16, Appendix C].

\section{THE SYMmetric GAUSSIAN C-RAN}

First, we specialize Theorem 1 to the symmetric Gaussian C-RAN defined in (6)-(7) where $P_{m}=P$ for all $m=$ $1, \ldots, M$. Choose $\left(X_{1}, \ldots, X_{M}\right)$ to be jointly Gaussian with the covariance matrix

$$
\mathbf{K}_{M}(\rho)=\left[\begin{array}{cccc}
P & \rho P & \ldots & \rho P \\
\rho P & P & \ldots & \rho P \\
\vdots & & \ddots & \vdots \\
\rho P & \ldots & \rho P & P
\end{array}\right]
$$

Remark 2. Choosing $\left(X_{1}, \ldots, X_{M}\right)$ to be jointly Gaussian (and/or symmetric) is not necessarily optimal for (13)-(14), but it gives a lower bound on the capacity.

Theorem 3. The rate $R$ is achievable if it satisfies the following constraints for some non-negative parameter $\rho, 0 \leq \rho \leq 1$ :

$$
\begin{aligned}
& R \leq M C-\frac{1}{2} \log \left(\frac{P^{M}}{\operatorname{det}\left(\mathbf{K}_{M}(\rho)\right)}\right) \\
& R \leq \frac{1}{2} \log (1+P M(1+(M-1) \rho)) .
\end{aligned}
$$

Remark 3. One can recursively calculate $\operatorname{det}\left(\mathbf{K}_{M}(\rho)\right)$ :

$$
\begin{aligned}
\operatorname{det}\left(\mathbf{K}_{M}(\rho)\right) & =P^{M}\left(1-\rho^{2} \sum_{i=1}^{M-1} i(1-\rho)^{i-1}\right) \\
& =P^{M}(1-\rho)^{M-1}(1+(M-1) \rho) .
\end{aligned}
$$

Let $R^{(\ell)}$ be the maximum achievable rate given by (27)(28). The RHS of (27) is non-increasing in $\rho$ and the RHS of (28) is increasing in $\rho$. Therefore, we have the following two cases for the optimizing solution $\rho^{(\ell)}$ :

- If $M C \leq \frac{1}{2} \log (1+P M)$ then

$$
\rho^{(\ell)}=0 \quad \text { and } \quad R^{(\ell)}=M C .
$$

- Otherwise, $\rho^{(\ell)}$ is the unique solution of $\rho$ in

$$
\begin{aligned}
& \frac{1}{2} \log (1+P M(1+(M-1) \rho)) \\
& \quad=M C-\frac{1}{2} \log \left(\frac{1}{(1-\rho)^{M-1}(1+(M-1) \rho)}\right)
\end{aligned}
$$

and we have

$$
R^{(\ell)}=\frac{1}{2} \log \left(1+P M\left(1+(M-1) \rho^{(\ell)}\right)\right) .
$$

We next specialize Theorem 2 to symmetric Gaussian CRANs.

Theorem 4. The rate $R$ is achievable only if there exists $\rho$, $0 \leq \rho \leq 1$, such that the following inequalities hold for all $N \geq 0$ :

$$
\begin{aligned}
R \leq & M C-(M-1) \frac{1}{2} \log \left(2^{2 R}+N\right)-\frac{1}{2} \log (1+N) \\
& +\frac{M}{2} \log (1+N+P((M-1)(1-\rho)(1+(M-1) \rho))) \\
R \leq & \frac{1}{2} \log (1+P M(1+(M-1) \rho) .
\end{aligned}
$$

Proof of Theorem 4. Set

$$
U_{i}=Y_{i}+Z_{N, i}, \quad i=1, \ldots, n
$$

where $\left\{Z_{N, i}\right\}_{i=1}^{n}$ are identically distributed according to the normal distribution $\mathcal{N}(0, N)$ and are independent from each other and $X_{1}^{n}, \ldots, X_{M}^{n}$. The variance $N$ is to be optimized.

In order to find a computable upper bound in (19), we need to lower bound $h(U \mid Q)$. Recall that $U$ is a noisy version of 
$Y$. We thus use the conditional entropy power inequality [18, Theorem 17.7.3]:

$$
\begin{aligned}
h(U \mid Q) & \geq \frac{1}{2} \log \left(2^{2 h(Y \mid Q)}+2 \pi e N\right) \\
& \geq \frac{1}{2} \log \left(2 \pi e\left(2^{2 R}+N\right)\right) .
\end{aligned}
$$

Substituting (36) into the first constraint of (19), we obtain:

$$
\begin{aligned}
R \leq & M C-(M-1) \frac{1}{2} \log \left(2 \pi e\left(2^{2 R}+N\right)\right) \\
& +\sum_{m=1}^{M} h\left(U \mid X_{m} Q\right)-h\left(U \mid X_{\mathcal{M}}\right) \\
\leq & M C-(M-1) \frac{1}{2} \log \left(2 \pi e\left(2^{2 R}+N\right)\right) \\
& +\sum_{m=1}^{M} h\left(U \mid X_{m}\right)-h\left(U \mid X_{\mathcal{M}}\right) .
\end{aligned}
$$

Now consider the second term in (19) with $\mathcal{S}=\varnothing$ :

$$
\begin{aligned}
R & \leq I\left(X_{\mathcal{M}} ; Y \mid Q\right) \\
& \leq I\left(X_{\mathcal{M}} ; Y\right) \\
& =h(Y)-h\left(Y \mid X_{\mathcal{M}}\right)
\end{aligned}
$$

Note that the RHSs of (37), (38) are both concave in $p\left(x_{\mathcal{M}}\right)$ and symmetric with respect to $X_{1}, \ldots, X_{M}$. Therefore, a symmetric $p\left(x_{\mathcal{M}}\right)$ maximizes them. Let $\mathbf{K}$ denote the covariance matrix of an optimal symmetric solution. We have

$$
\mathbf{K}_{i i}=P, \quad \mathbf{K}_{i j}=P \rho .
$$

Using the conditional version of the maximum entropy lemma [19], we can upper bound the differential entropies that appear with positive sign in (37) and (38) by their Gaussian counterparts, and $h\left(U \mid X_{\mathcal{M}}\right)$ and $h\left(Y \mid X_{\mathcal{M}}\right)$ can be written explicitly because the channels from $X_{\mathcal{M}}$ to $U$ and $Y$ are Gaussian. We obtain (33) and (34). Note that the RHSs of both bounds are increasing in $P$ and therefore there is no loss of generality in choosing $\mathbf{K}_{i i}=P\left(\right.$ among $\left.\mathbf{K}_{i i} \leq P\right)$.

The upper bound of Theorem 4 and the lower bound of Theorem 3 are plotted in Fig. 2 for $M=3$ and $P=1$, and they are compared with the lower bounds of message sharing [4], and compression [5]. One sees that our lower and upper bounds are close and they match over a wide range of $C$. Moreover, establishing partial cooperation among the relays through Marton's coding offers significant gains. Fig. 3 plots the capacity bounds for $P=1$ and different values of $M$.

We next compare the lower and upper bounds. Let

$$
\begin{aligned}
C_{C} & =\frac{1}{2 M} \log (1+P M) \\
C_{L} & =\frac{1}{2 M} \log \left(\frac{1+\frac{M^{2}}{2} P}{\left(\frac{M}{2(M-1)}\right)^{M-1} \frac{M}{2}}\right) \\
C_{U} & =\frac{1}{2 M} \log \left(\frac{1+M P\left(1+(M-1) \rho^{(2)}\right)}{\left(1-\rho^{(2)}\right)^{M-1}\left(1+(M-1) \rho^{(2)}\right)}\right)
\end{aligned}
$$

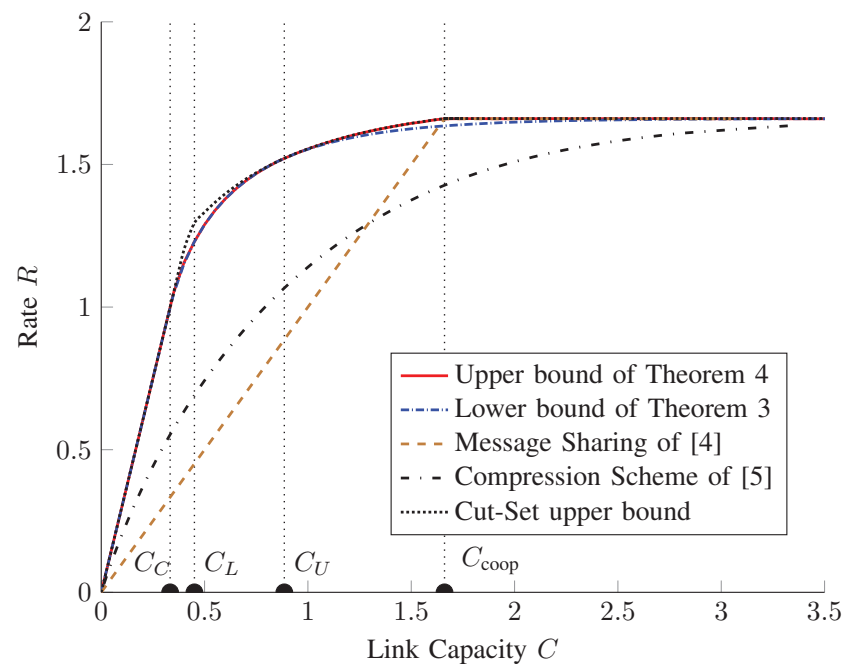

Fig. 2: Capacity bounds as functions of $C(M=3, P=1)$.

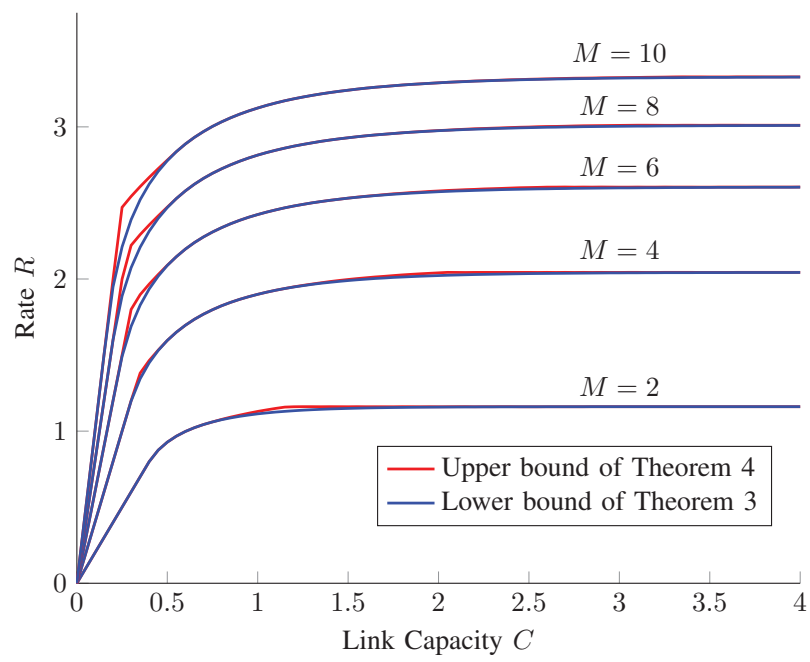

Fig. 3: Capacity bounds as functions of $C$ for $P=1$ and different values of $M$.

where

$$
\rho^{(2)}=\frac{M-2-\frac{1}{P}+\sqrt{\left(M-2-\frac{1}{P}\right)^{2}+4(M-1)}}{2(M-1)} .
$$

Theorem 5. The lower bound of Theorem 3 matches the upper bound of Theorem 4 if

$$
C \leq C_{C}
$$

or if

$$
C_{L} \leq C \leq C_{U}
$$

where $C_{C}, C_{L}, C_{U}$ are defined in (40)-(42).

Remark 4. Theorem 5 recovers [12, Theorem 5] for $M=2$.

Remark 5. For $C \leq C_{C}$, no cooperation is needed among the transmitters and the capacity is equal to $M C$. 
Remark 6. For $C$ large enough, full cooperation is possible through superposition coding and the capacity is

$$
C^{(M)}=\frac{1}{2} \log \left(1+M^{2} P\right), \quad C \geq C_{\text {coop }}
$$

where

$$
C_{\text {coop }}=\frac{1}{2} \log \left(1+M^{2} P\right) .
$$

The rate (46) is not achievable by Theorem 3 except when $C \rightarrow \infty$. This rate is achievable by message sharing.

Proof of Theorem 5. To find regimes of $P$ and $C$ for which the lower and upper bounds match, we mimic the analysis in [12, Appendix F]. Consider the lower bound in Theorem 3, and in particular its maximum achievable rate $R^{(\ell)}$ which is attained by $\rho^{(\ell)}$, see (30)-(32). If (44) holds, we have $\rho^{(\ell)}=0, R^{(\ell)}=M C$, and thus the cut-set bound is achieved. Otherwise, we proceed as follows.

Consider (33). Since $R \geq R^{(\ell)}$, and using the definition of $R^{(\ell)}$ in (32), we can further upper bound (33) and obtain

$$
\begin{aligned}
R \leq & M C-(M-1) \frac{1}{2} \log \left(1+N+P M\left(1+(M-1) \rho^{(\ell)}\right)\right. \\
& +\frac{M}{2} \log (1+N+P((M-1)(1-\rho)(1+(M-1) \rho))) \\
& -\frac{1}{2} \log (1+N) .
\end{aligned}
$$

Call the RHS of (48) $g_{1}(\rho)$ and the RHS of (34) $g_{2}(\rho)$. Fix $N$ as a function of $\rho^{(\ell)}$ such that

$$
I^{\left(G, \rho^{(\ell)}\right)}\left(X_{\mathcal{M}} \mid U\right)=0
$$

where $I^{\left(G, \rho^{(\ell)}\right)}\left(X_{\mathcal{M}} \mid U\right)$ is $I\left(X_{\mathcal{M}} \mid U\right)$ evaluated for a fully symmetric Gaussian distribution with correlation factor $\rho^{(\ell)}$. One can verify that the following choice of $N$ satisfies (49):

$$
N=P \frac{\left(1-\rho^{(\ell)}\right)\left(1+(M-1) \rho^{(\ell)}\right)}{\rho^{(\ell)}}-1 .
$$

The right inequality in (45) ensures $N \geq 0$.

With this choice of $N, g_{1}(\rho)$ is exactly equal to

$$
M C-I^{\left(G, \rho^{(\ell)}\right)}\left(X_{\mathcal{M}}\right)
$$

at $\rho=\rho^{(\ell)}$. Note that $\rho^{(\ell)}$ is defined in (31), and thus $g_{1}(\rho)$ crosses $g_{2}(\rho)$ at $\rho^{(\ell)}$. Since $g_{2}(\rho)$ is increasing in $\rho$, the maximum admissible rate by (34) and (48) matches $R^{(\ell)}$ if $g_{1}(\rho)$ is non-increasing for $\rho \geq \rho^{(\ell)}$. This is ensured by the left inequality in (45).

\section{CONCLUding REMARKS}

We studied the downlink of symmetric C-RANs with multiple relays and a single receiver, and established lower and upper bounds on the capacity. The lower bound uses Marton's coding to establish partial cooperation among the relays and improves on schemes that are based on message sharing and compression for scalar Gaussian C-RANs (see Fig. 2). The upper bound generalizes the bounding techniques of [11], [12]. When specialized to symmetric Gaussian C-RANs, the lower and upper bounds meet over a wide range of $C$ and this range gets large as $M$ and/or $P$ get large.

\section{REFERENCES}

[1] S. H. Park, O. Simeone, O. Sahin, and S. Shamai, "Fronthaul compression for cloud radio access networks: Signal processing advances inspired by network information theory," IEEE Signal Proc. Mag., vol. 31, no. 6, pp. 69-79, Nov 2014.

[2] W. Yu, "Cloud radio-access networks: Coding strategies, capacity analysis, and optimization techniques," in Commun. Theory Workshop, May 2016, plenary Talk.

[3] M. Peng, C. Wang, V. Lau, and H. V. Poor, "Fronthaul-constrained cloud radio access networks: insights and challenges," IEEE Wireless Commun., vol. 22, no. 2, pp. 152-160, April 2015.

[4] B. Dai and W. Yu, "Sparse beamforming and user-centric clustering for downlink cloud radio access network," IEEE Access, vol. 2, pp. 13261339, 2014.

[5] S. H. Park, O. Simeone, O. Sahin, and S. Shamai, "Joint precoding and multivariate backhaul compression for the downlink of cloud radio access networks," IEEE Trans. Signal Proc., vol. 61, no. 22, pp. 56465658, Nov 2013.

[6] P. Patil and W. Yu, "Hybrid compression and message-sharing strategy for the downlink cloud radio-access network," in Inf. Theory and Appl. Workshop, Feb 2014, pp. 1-6.

[7] N. Liu and W. Kang, "A new achievability scheme for downlink multicell processing with finite backhaul capacity," in IEEE Int. Symp. Inf. Theory, June 2014, pp. 1006-1010.

[8] C. Wang, M. A. Wigger, and A. Zaidi, "On achievability for downlink cloud radio access networks with base station cooperation," CoRR, vol. abs/1610.09407, 2016. [Online]. Available: http://arxiv.org/abs/1610.09407

[9] T. Yang, N. Liu, W. Kang, and S. Shamai, "An upper bound on the sum capacity of the downlink multicell processing with finite backhaul capacity," CoRR, vol. abs/1609.00833, 2016. [Online]. Available: http://arxiv.org/abs/1609.00833

[10] D. Traskov and G. Kramer, "Reliable communication in networks with multi-access interference," in Proc. Inf. Theory Workshop, Tahoe City, CA, Sept. 2007, pp. 343-348.

[11] W. Kang, N. Liu, and W. Chong, "The Gaussian multiple access diamond channel," IEEE Trans. Inf. Theory, vol. 61, no. 11, pp. 6049-6059, Nov 2015.

[12] S. Saeedi Bidokhti and G. Kramer, "Capacity bounds for diamond networks with an orthogonal broadcast channel," IEEE Trans. Inf. Theory, vol. 62, no. 12, pp. 7103-7122, Dec 2016.

[13] A. El Gamal and Y. H. Kim, Network Information Theory. Cambridge, UK: Cambridge University Press, 2011.

[14] R. Venkataramani, G. Kramer, and V. K. Goyal, "Multiple description coding with many channels," IEEE Trans. Inf. Theory, vol. 49, no. 9, pp. 2106-2114, Sep. 2006.

[15] S. Saeedi Bidokhti and G. Kramer, "Capacity bounds for a class of diamond networks," in Proc. Int. Symp. Inf. Theory, Honolulu, HI, July 2014, pp. 1196-1200.

[16] S. Saeedi Bidokhti, G. Kramer, and S. Shamai, "Capacity bounds on the downlink of symmetric, multi-relay, single receiver C-RAN networks," 2017. [Online]. Available: http://stanford.edu/ saeedi/cran.pdf

[17] L. Ozarow, "On a source-coding problem with two channels and three receivers," Bell Sys. Techn. J., vol. 59, no. 10, pp. 1909-1921, Dec. 1980.

[18] T. M. Cover and J. A. Thomas, Elements of Information Theory. New York, NY, USA: Wiley-Interscience, 2006.

[19] J. Thomas, "Feedback can at most double Gaussian multiple access channel capacity," IEEE Trans. Inf. Theory, vol. 33, no. 5, pp. 711-716, Sept. 1987. 